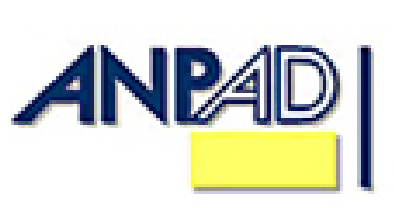

Disponível em http://www.anpad.org.br/rac

RAC, Curitiba, v. 14, n. 2, art. 5, pp. 268-288, Mar./Abr. 2010

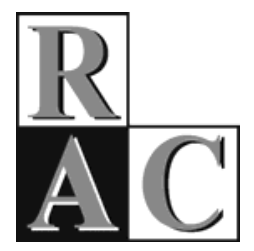

\title{
Publicação Científica Nacional e Internacional sobre Franchising: Levantamento e Análise do Período 1998 - 2007
}

\author{
National and International Scientific Publication about Franchising: Analysis 1998- \\ 2007
}

Pedro Lucas de Resende Melo * Doutorando em Administração pela FEA-USP. Professor da FEA/PUC-SP, São Paulo/SP, Brasil.

Tales Andreassi Doutor em Administração pela FEA-USP. Professor da EAESP-FGV, São Paulo/SP, Brasil.

* Endereço: Pedro Lucas de Resende Melo

Rua Monte Alegre, 984, Perdizes, São Paulo/SP. E-mail: plresende@pucsp.br

Copyright (C 2010 RAC. Todos os direitos, inclusive de tradução, são reservados. É permitido citar parte de artigos sem autorização prévia desde que seja identificada a fonte. 


\title{
RESUMO
}

O sistema de franquia movimenta na economia brasileira cerca de R\$ 46 bilhões/ano, por meio de 1.197 redes de franquias que licenciam 65.500 unidades, gerando quase 600 mil empregos diretos. Nos EUA esse número é ainda mais significativo, com cerca de 760.000 unidades franqueadas e movimentam US\$ 1,53 trilhão/ano na economia. O objetivo deste trabalho é desenvolver uma inédita meta-análise sobre franchising, envolvendo 61 artigos científicos nacionais e internacionais publicados de 1998 a 2007 e veiculados à Anpad e em periódicos relacionados no Proquest (Qualis A, B ou C). São abordados os seguintes questionamentos: como tem evoluído a pesquisa científica em franchising; em quais veículos acadêmicos estão vinculados; as principais temáticas abordadas; os vínculos institucionais destes autores; a participação dos estados e países; a predominância de determinados autores; os métodos de pesquisa utilizados e os segmentos econômicos de aplicação. Os resultados evidenciam a concentração dos trabalhos nacionais no Enanpad; a predominância de trabalhos sobre Empreendedorismo e Estratégia, internacionalmente, com foco em teorias de econômicas; a maioria dos autores oriundos de instituições do sul e sudeste do Brasil e dos EUA; uma forte predominância de pesquisas e a aplicação das pesquisas em setores diversos da economia.

Palavras-chave: franchising; meta-análise; empreendedorismo.

\begin{abstract}
The franchising system generates about forty-six billion reais a year in the Brazilian economy by way of 1,197 franchising networks that licnse 65,500 units, generating almost 600,000 direct jobs. In the USA that number is higher still, with around 760,000 units that generate 1.53 trillion dollars a year in the economy. The objective of this paper is to develop an unpublished goal-analysis on franchising involving 61 Brazilian and international scientific papers published from 1998 to 2007 and published by Anpad and in related periodical in Proquest (Qualis A, B or C). Saint approached the following questions: how scientific research into franchises has developed; in which academic vehicles are they linked; the main themes that are dealt with; which institutions these authors are connected with; the participation of states and countries; the predominance of certain authors; the research methods used and the economic segments of application. The results show that Brazilian works are concentrated in Enanpad; the predominance of works on Entrepreneurship and Strategy, internationally, with focus in economic theories; most of the authors are connected to institutions in the south and south-east of Brazil and the USA; a strong predominance of the surveys and the application of the research in several sections of the economy.
\end{abstract}

Key words: franchising; meta-analysis; entrepreneurship. 


\section{INTRODUÇÃO}

O sistema de franchising se baseia numa relação contratual referente a uma franquia que é desenvolvida entre duas partes: o franqueado e o franqueador. Dessa forma, o franchising é uma alternativa à diversificação que pode ser considerada uma estratégia cooperativa em que as firmas adotam para dispersar os riscos e compartilhar competências (Shane, 2005). Franquia, segundo uma definição formal,

é um acordo contratual entre duas companhias legalmente independentes em que o franqueador concede ao franqueado o direito de vender o produto do franqueador ou fazer negócios utilizando sua marca registrada em determinado local durante um intervalo de tempo especificado (Hitt, Ireland, \& Hoskisson, 2002, p. 380).

Este modelo de negócios tem dentre suas principais finalidades o estabelecimento de estratégias para a distribuição e comercialização de produtos e serviços desenvolvidos, que são produzidos pelo franqueador e comercializados em diversas localidades pelos franqueados (Dornelas, 2001; Hisrich \& Peters, 2004).

O franchising pode ser considerado um segmento do empreendedorismo, servindo como alternativa ao empreendedor que deseja estabelecer-se com marcas e know-how consolidados pelos franqueadores, o que contribui para reduzir as incertezas ao se abrir um negócio (Correa, Hoeltegebaum, \& Machado, 2006; Luiz, Motoki, Vilela, Ura, \& Lourenzani, 2006). Outros benefícios também podem ser identificados nas franquias, como os ganhos de escala, devido ao maior acesso tecnológico possibilitado pelo franqueador; a maior facilidade de obter financiamentos para expansão do empreendimento; menor dispêndio de recursos para o monitoramento e o controle das unidades, quando comparado às lojas próprias (Azevedo \& Silva, 2003).

Luiz et al. (2006) consideram que as vantagens do sistema de franchising são superiores aos negócios independentes, principalmente por fornecerem ao empreendedor um modelo de gestão em parceria, em que há maior troca de informações e práticas inovadoras nos mercados em que a rede de franchising atua.

É marcante a opção estratégica pelo sistema de franchising, tornando-se cada vez mais disseminado em escalas globais, sendo responsável por $40 \%$ do volume de venda a varejo nos Estados Unidos, 32\% na Grã-Bretanha, 25\% na Austrália e 24\% no Brasil (Melo, Borini, \& Ribeiro, 2007; Swartz, 2001).

Os dados do mercado mundial de franquias demonstram que os Estados Unidos dominam a adoção do franchising internacionalmente, com mais de 760.000 unidades franqueadas, gerando 18 milhões de empregos e movimentando US\$ 1,53 trilhão anualmente na economia; em segundo e terceiro lugares em número de franquias seguem, respectivamente, o Canadá e a Coréia do Sul. O Brasil destaca-se por ser o quarto país em número de franquias com 65.500 unidades franqueadas por 1.197 redes, o que representa uma geração de cerca de 600 mil empregos diretos e estima-se em quase 2 milhões de empregos indiretos (Associação Brasileira de Franchising [ABF], 2008; International Franchise Association [IFA], 2008; Rizzo, 2005).

O desenvolvimento do franchising no Brasil tem ocorrido com grande vigor nos últimos anos, em especial a partir da criação da Associação Brasileira de Franchising [ABF] em 1987. Os fatos determinantes para a propagação das redes de franquias no Brasil foram a criação e expansão de shopping centers; o desenvolvimento dos centros urbanos; além de um destacado atrativo para os empreendedores, a baixa mortalidade destes empreendimentos. Em empreendimentos maduros, com dez anos de existência, a taxa de mortalidade das franquias é próxima a 9\%, enquanto em negócios independentes este índice é de aproximadamente 80\% (Rizzo, 2005).

Segundo os dados da ABF, em 2007 as redes de franquia brasileiras movimentaram R 46 bilhões e obtiveram uma média de crescimento no faturamento de aproximadamente $16 \%$, valor bem superior 
ao da economia brasileira para este período, que se estabeleceu em 5,4\%. O destaque no franchising tem sido para os setores de alimentação, educação e treinamento, automotivo, informática e eletrônicos (ABF, 2008; Canzian \& Lage, 2008).

Tendo caracterizado a relevância do franchising tanto nacional como internacionalmente, surgem alguns questionamentos de pesquisa: (i) como tem evoluído a pesquisa científica em franchising; (ii) em quais veículos acadêmicos estas publicações estão vinculadas; (iii) quais as principais temáticas abordadas; (iv) os vínculos institucionais destes autores; (v) a participação dos estados brasileiros e países nestas produções; (vi) a predominância de determinados autores; (vii) os métodos de pesquisa utilizados; e (viii) os segmentos econômicos de aplicação destes estudos. A busca por estas respostas passa necessariamente pela análise de um universo de trabalhos científicos representativos da pesquisa sobre franchising no Brasil e no exterior.

Com isso o objetivo principal deste trabalho é traçar o perfil da pesquisa científica sobre franchising no Brasil e no exterior. Para tanto foram utilizadas publicações dos eventos e periódicos coordenados pela Anpad, disponibilizados em seu website, como também periódicos internacionais classificados como A, B ou C pelo sistema Qualis e disponíveis no portal virtual Proquest. O período de análise figura-se entre 1998 e 2007, visando analisar os últimos 10 anos de produção científica sobre franchising.

Estudos deste tipo são conhecidos como meta-análises. Mesmo não sendo raros no meio acadêmico, não houve nenhum registro desse tipo de estudo sobre franchising, o que lhe confere caráter de ineditismo. A sua importância reside em expectativas de contribuições para a evolução das pesquisas sobre o sistema de franchising brasileiro ao realizar uma comparação deste desenvolvimento em relação ao exterior, principalmente com os Estados Unidos, por ser esse o país em que há maior expertise no franchising.

Este trabalho se inicia com um resgate teórico de estudos que envolvem meta-análises em vários campos da Administração. A seguir, apresentam-se e discutem-se os dados encontrados. Por fim, conclui-se este estudo com recomendações e críticas referentes à pesquisa científica nacional e internacional sobre franchising.

\section{RESGATE TEÓRICO}

É possível recorrer a alguns artigos que tratam da análise de revisões de publicações (meta-análises), como é o caso de Combs e Ketchen (2003), ao investigarem publicações que tratam das razões que levam os empreendedores a optarem pelo franchising, tendo os autores utilizado a base de dados EconLit entre 1970 e 2001. Dant, Paswan e Kaufman (1996) também exploram em sua pesquisa o setor de franchising, ao selecionarem publicações que tratam do redirecionamento de propriedades em periódicos da década de 90.

Com relação aos estudos mercadológicos, pode-se notar diversos trabalhos deste caráter realizados nos últimos anos, como é o caso de Szymanski, Bharadwaj e Varadarajan (1993), que avaliam a relação da participação de mercado e a lucratividade de empresas entre os anos de 1970 e 1991. Geyskens, Steenkamp e Kumar (1999) procuram testar a confiança e os compromissos dos canais mercadológicos em publicações entre os anos de 1970 e 1996.

Por sua vez, a área de contabilidade e finanças é representada com algumas meta-análises, como Horngren (1989), que trata especificamente das mudanças na contabilidade de custos em um período de 32 anos. Vollmers (1996) agrega estudos nesta mesma área entre os anos de 1920 e 1950 nos Estados Unidos. Já Shields (1997) analisa 152 publicações em contabilidade gerencial. Otchere (2003) trata da análise evolutiva dos trabalhos em contabilidade e finanças.

Nacionalmente, pode-se notar diversas meta-análises atreladas aos eventos da Anpad, a começar por Wood e Chueke (2007), que retratam a produção científica em diversas áreas da administração e criam 
uma classificação de produtividade nacional, utilizando para isto revistas nacionais A no período de 2002 a 2006.

Rossoni, Guarido, Franscisconi e Albuquerque (2007) analisaram artigos sobre estratégia nos anais do Enanpad e 3E'S no período de 2001 a 2006. Por sua vez, Silva e Campos (2007) investigaram a produção científica na área de gestão internacional por meio dos periódicos nacionais classificados com o conceito A e os artigos publicados no Enanpad entre 1997 e 2006.

A área de marketing é representada por um estudo de Vieira (1998) sobre a influência das publicações desta área no Enanpad, ao utilizar como referência os anos compreendidos entre 1990 e 1997. Já Almeida, Lopes e Pereira (2006) fizeram seu estudo em publicações sobre marketing de relacionamento em periódicos brasileiros entre 1990 e 2004.

A área de recursos humanos apresenta um meta-estudo que envolve artigos aceitos no Enanpad na década 90 (Caldas, Tonelli, \& Lacombe, 2002). Hocayen-da-Silva, Rossoni e Ferreira (2006) apresentam um quadro geral da produção científica em administração pública e gestão social, tendo como base os anais do Enanpad entre 2000 e 2005.

Diversos estudos são apresentados sobre a área de sistemas de informação, como nos confere Hoppen et al. (1998), ao apresentarem uma avaliação de publicações em periódicos sobre sistemas de informação entre 1990 e 1997. Rossoni e Hocayen-da-Silva (2007) acrescentam as suas contribuições à administração da informação por meio do levantamento de publicações nos anais do Enanpad no período de 2001 a 2006. Com temática próxima da última pesquisa mencionada, Rossoni, Ferreira e Hocayen-da-Silva (2006) traçam um panorama da produção científica em administração de ciência e tecnologia, valendo-se dos anais do Enanpad e do Simpósio de Gestão da Inovação e Tecnológica de 2000 a 2005. Zimmer e Leis (2007) vão adiante em um trabalho que envolve a análise de artigos sobre gestão do conhecimento em periódicos classificados como Qualis A e presentes nos anais do Enanpad no período de 1997 a 2006. Por sua vez, Durante e Maurer (2007) prosseguem com estudos sobre gestão do conhecimento e gestão da informação, ao contemplarem as publicações presentes no banco de teses da Capes, nos anais do Enanpad e em revistas científicas de 2000 a 2005.

Finalmente, Closs, Aramburú e Antunes (2006) investigam artigos publicados na área de ensino e pesquisa de administração em uma avaliação que envolve o enfoque do paradigma da complexidade do Enanpad nos anos de 2002 a 2005. Matias, Machado, Machado e Martins (2007) representam a área de finanças, analisando trabalhos apresentados no Congresso de Controladoria e Contabilidade da USP em 2006.

\section{METODOLOGIA}

Esta pesquisa pretendeu analisar o perfil das publicações científicas nacionais e internacionais que tratam do sistema de franchising. Para isto, alguns critérios foram previamente definidos, a fim de se delimitar o universo a ser pesquisado.

Primeiramente, quanto ao período de publicação, estes trabalhos deveriam ter sido publicados entre os anos de 1998-2007, o que corresponde aos últimos dez anos. Adotou-se este critério temporal, visando dar um caráter recente ao trabalho, a fim de se debater e visualizar caminhos para futuras pesquisas sobre o sistema de franchising.

Quanto à coleta de dados, dividiu-se a amostra em duas áreas, as publicações nacionais e as internacionais. Nas publicações nacionais, foi estabelecido que somente fizessem parte deste estudo artigos vinculados ao website da Associação Nacional de Pós-Graduação e Pesquisa em Administração [ANPAD] (Recuperado em 30 outubro, 2007, de http://www.anpad.org.br); nisto incluem-se os periódicos Revista de Administração de Empresas [RAE], Revista de Administração Contemporânea 
[RAC], Brazilian Administration Review [BAR], Revista de Administração Contemporânea Eletrônica [RAC-E] e os eventos Encontro da Anpad [ENANPAD], Encontro de Marketing [EMA], Encontro de Estudos Organizacionais [ENEO], Encontro de Estudos em Estratégia [3E’S], Simpósio de Gestão da Inovação Tecnológica [Simpósio], Encontro de Administração Pública e Governança [ENAPG], Encontro de Gestão de Pessoas e Relações do Trabalho [ENGPR], Encontro de Administração da Informação [ENADI] e Encontro de Ensino e Pesquisa em Administração e Contabilidade [ENEPQ]. Esta escolha se deu devido a uma maior conveniência de acesso à base de dados destes periódicos e anais e, principalmente, por sua avaliação perante o sistema Qualis, no qual tais periódicos possuem classificação entre Nacional A e Internacional B, e os eventos entre Nacional A e Internacional A. Entretanto somente foram encontrados artigos no perfil estabelecido nos seguintes veículos científicos: ENANPAD (19); 3E’S (2); EMA (1); Simpósio (1) e RAC (1), totalizando 24 publicações.

Nos trabalhos internacionais utilizou-se como base de busca o portal virtual Proquest (Recuperado em 15 outubro, 2007, de http://www.proquest.com), por ser um aglutinador de periódicos internacionais. Para estes trabalhos internacionais estabeleceu-se o critério da presença destes periódicos na tabela Qualis (2007), que titula tais publicações em Internacional A, B ou C. Dados os critérios estabelecidos, foram localizados 37 artigos presentes em quinze periódicos (Tabela 3).

Posteriormente à seleção destes artigos, procedeu-se à leitura dos seus resumos, problematização, metodologia e conclusões. Em casos específicos, houve a leitura integral de seus textos, na busca de uma compreensão maior do assunto tratado. Os estudos de Cardoso, Pereira e Guerreiro (2007); Tonelli, Caldas, Lacombe e Tinoco (2003); Vieira (1998) e Machado-da-Silva, Cunha e Amboni (1990) subsidiaram a elaboração dos índices utilizados nesta pesquisa, sendo estes: (i) artigos publicados nacionalmente e internacionalmente; (ii) classificação segundo a Tabela Qualis; (iii) local de publicação; (iv) período de publicação; (v) temáticas abordadas; (vi) filiação dos autores; (v) participação dos estados brasileiros e países; (vi) autores por artigos; (vii) publicação por pesquisador; (viii) método de pesquisa utilizado e (ix) segmento econômico de aplicação.

\section{APRESENTAÇÃO E ANÁlISE dOS RESULTADOS}

A exposição e discussão dos dados encontrados nesta pesquisa serão apresentadas em seguida. Para cada categoria, haverá uma comparação entre os indicadores nacionais e internacionais.

\section{Estudos Nacionais e Internacionais}

Foram obtidos 24 estudos nacionais, o que corresponde a cerca de $40 \%$ da amostra analisada, e 37 artigos internacionais, perfazendo os outros $60 \%$ dos artigos sobre franchising, que preencheram os requisitos listados na metodologia. Esta diferença pode ser justificada por dois argumentos, tanto devido a uma maior base de trabalhos internacionais, veiculados no Proquest, do que nacionalmente pela Anpad, como também por um maior número de estudos sobre franchising em outros países, principalmente os Estados Unidos e países da comunidade européia.

Tabela 1

\section{Estudos Nacionais e Internacionais}

\begin{tabular}{lll}
\hline ORIGEM & PARTICIPAÇÃO & \% \\
\hline Nacional & 24 & 39,3 \\
Internacional & 37 & 60,6 \\
TOTAL & $\mathbf{6 1}$ & $\mathbf{1 0 0 , 0}$ \\
\hline
\end{tabular}

Nota. Fonte: elaborado pelos autores. 


\section{Tabela Qualis}

Dentre os critérios adotados neste estudo, quanto à produção científica sobre franchising, foi estabelecido que para figurar entre os estudos nacionais, era imprescindível que o trabalho estivesse vinculado à Anpad. Da amostra estudada, aproximadamente $96 \%$ são trabalhos vinculados a eventos Internacionais A ou Nacionais A, sendo encontrado somente um artigo publicado em periódicos, no caso Nacional A.

Por sua vez, dentre os estudos internacionais, para sua participação foi necessário o periódico estar listado na tabela Qualis (2007) como Internacional A, B ou C. Constatou-se que 78\% dos artigos internacionais sobre franchising estão classificados como A ou B, o que gera maior reconhecimento por estes trabalhos analisados.

Esta metodologia visou selecionar artigos, tanto nacionais quanto internacionais, que tenham sua qualidade atestada por instituições que adotam rigorosos padrões de qualidade em suas avaliações.

Tabela 2

\section{Tabela Qualis}

\begin{tabular}{lll}
\hline CLASSIFICAÇÃO & PARTICIPAÇÃO & $\mathbf{\%}$ \\
\hline NACIONAL & & \\
Eventos - Internacional A & 19 & 79,1 \\
Eventos - Nacional A & 04 & 16,6 \\
Periódico - Nacional A & 01 & 4,1 \\
TOTAL & $\mathbf{2 4}$ & $\mathbf{1 0 0 , 0}$ \\
\hline
\end{tabular}

\section{INTERNACIONAL}

\begin{tabular}{lll} 
Periódico - Internacional A & 13 & 35,1 \\
Periódico - Internacional B & 16 & 43,2 \\
Periódico - Internacional C & 08 & 21,6 \\
TOTAL & 37 & $\mathbf{1 0 0 , 0}$ \\
\hline
\end{tabular}

Nota. Fonte: elaborado pelos autores.

\section{Local de Publicação}

Em relação aos locais de publicação dos artigos nacionais publicados, cerca de $80 \%$ destes estão vinculados ao principal congresso da área, o Enanpad. Cumpre considerar neste item a natureza ainda recente dos demais congressos organizados pela Anpad, como o Encontro de Marketing (2003); o Encontro de Estudos de Estratégia (2005) e demais eventos temáticos; com isso, agregam-se poucos trabalhos em sua base.

Já nos estudos internacionais, três periódicos, Strategic Management Journal, European Journal of Marketing e Journal of Retailing concentram 43\% das publicações sobre franchising, estando o restante pulverizado em treze periódicos. 
Tabela 3

Local de Publicação

\begin{tabular}{llc}
\hline \multicolumn{1}{c}{ LOCAL } & PARTICIPAÇÃO & \% \\
\hline NACIONAL & & \\
Encontro da ANPAD (ENANPAD) & 19 & 79,1 \\
Encontro de Estudos de Estratégia (3E`S) & 02 & 8,3 \\
Encontro de Marketing (EMA) & 01 & 4,1 \\
Simpósio de Gestão da Inovação Tecnológica (Simpósio) & 01 & 4,1 \\
Revista de Administração Contemporânea (RAC) & 01 & 4,1 \\
TOTAL & $\mathbf{2 4}$ & $\mathbf{1 0 0}$ \\
\hline
\end{tabular}

INTERNACIONAL

Strategic Management Journal

$06 \quad 16,2$

$\begin{array}{lll}\text { European Journal of Marketing } & 06 & 16,2\end{array}$

$\begin{array}{lll}\text { Journal of Retailing } & 04 & 10,8\end{array}$

$\begin{array}{lll}\text { Journal of Management } & 03 & 8,1\end{array}$

$\begin{array}{lll}\text { Journal of Economic Behavior and Organization } & 03 & 8,1\end{array}$

$\begin{array}{lll}\text { Journal of Small Business and Enterprise Development } & 02 & 5,4\end{array}$

$\begin{array}{lll}\text { Journal of Small Business Management } & 02 & 5,4\end{array}$

$\begin{array}{lll}\text { Academy of Management Journal } & 02 & 5,4\end{array}$

$\begin{array}{lll}\text { Management Research News } & 02 & 5,4\end{array}$

$\begin{array}{lll}\text { European Business Review } & 01 & 2,7\end{array}$

$\begin{array}{lll}\text { Qualitative Market Research } & 01 & 2,7\end{array}$

$\begin{array}{lll}\text { Management Decision } & 01 & 2,7\end{array}$

$\begin{array}{lll}\text { Computer and Industrial Engineering } & 01 & 2,7\end{array}$

$\begin{array}{lll}\text { Journal of Business Ethics } & 01 & 2,7\end{array}$

$\begin{array}{lll}\text { The Academy of Management Review } & 01 & 2,7\end{array}$

$\begin{array}{lll}\text { TOTAL } & 37 & 100,0\end{array}$

Nota. Fonte: elaborado pelos autores.

\section{Período de Publicação}

Nos anos de 1998 e 2002 não houve nenhuma publicação sobre franchising em âmbito nacional nos veículos analisados. Em 2004 ocorreu o pico desta produção com 21\% dos artigos publicados, mesmo tendo reduzido a participação no ano subseqüente, $33 \%$ da produção estão concentrados nos dois últimos anos analisados, 2006 e 2007.

Internacionalmente os dados nos mostram que igualmente à ocorrência nacional, 2002 foi um ano em que não houve nenhuma publicação sobre franchising. O pico de produção, por sua vez, foi em 1999 , concentrando $24 \%$ da produção. Os últimos dois anos perfazem $16 \%$ da produção do período analisado. 


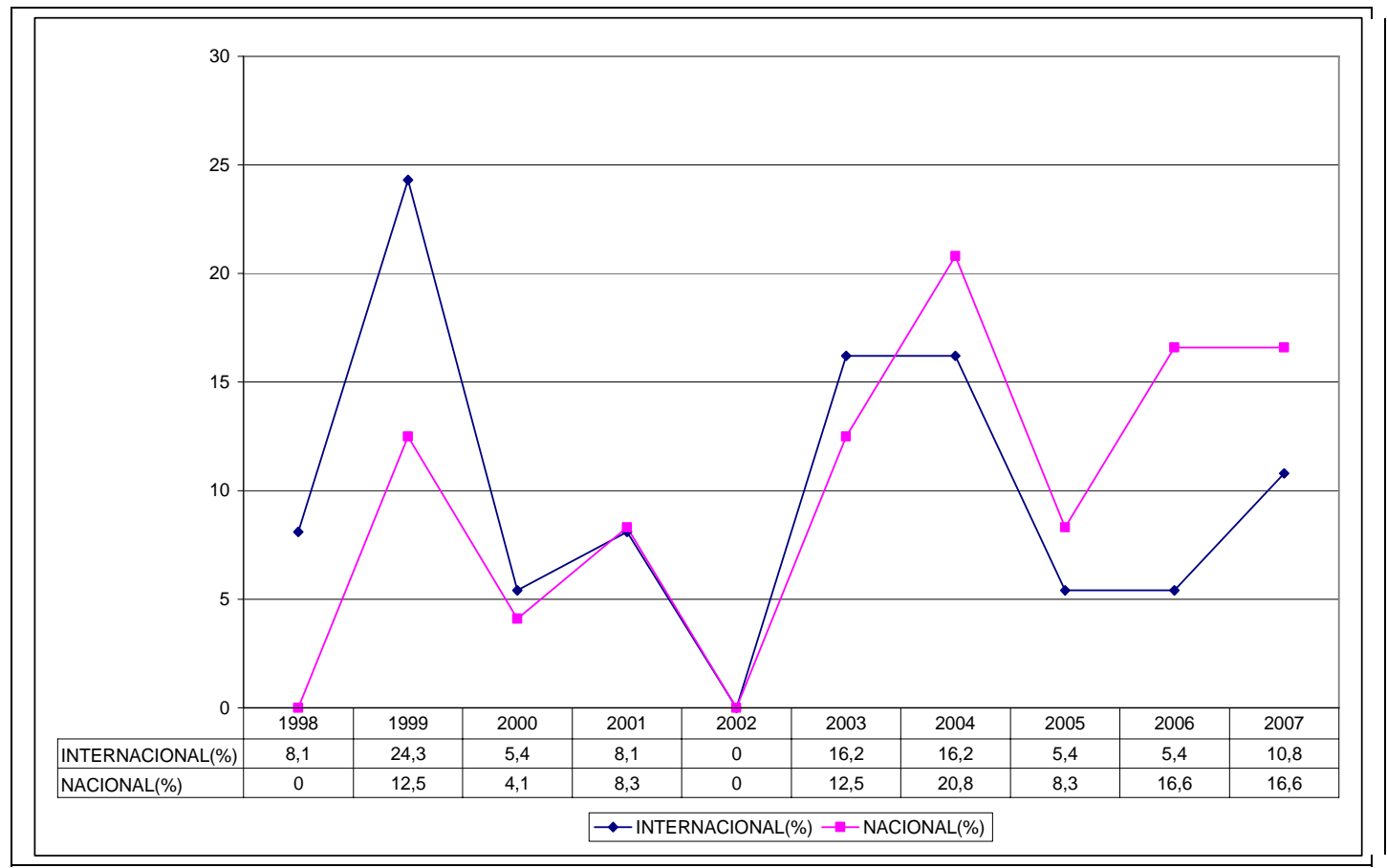

Figura 1. Período de Publicação

Fonte: elaborado pelos autores.

\section{Temáticas Abordadas}

Respondendo aos temas principais destes artigos, observa-se por parte dos estudos nacionais um destaque da temática de Empreendedorismo e Estratégia, com cinco trabalhos cada, o que corresponde a mais de $40 \%$ dos trabalhos nacionais. A justificativa para esta expressiva participação de artigos sobre Empreendedorismo que envolve franchising pode vir da organização do mercado de trabalho atual e de alternativas de investimento, em que empreendedores demonstram maior atração na criação de negócios por mediação do franchising, o que desperta a atenção dos pesquisadores para esta área. Os estudos relativos à Estratégia representam o caráter competitivo do franchising, em ambiente de transformações freqüentes. Vale ressaltar a busca pela internacionalização das franquias brasileiras, conferindo a terceira posição entre os trabalhos nacionais.

Entre os artigos internacionais, enfoques econômicos ganham destaque, sobretudo pela Teoria da Agência, presente em aproximadamente $25 \%$ dos artigos analisados, todos internacionais. Junto à Teoria da Agência, têm-se outras abordagens econômicas como a dos Custos de Transação, Resource Based View [RBV] e Escassez de Recursos, que juntamente com a Teoria da Agência constituem 38\% dos estudos internacionais analisados. Estudos de Estratégia ganham destaque também internacionalmente, em segundo lugar, revelando a preocupação pelas transformações dos mercados mundialmente. Por sua vez, não foi localizado nenhum trabalho que envolvesse esse Empreendedorismo como tema principal abordado. 
Tabela 4

\section{Principal Tema Abordado}

\begin{tabular}{lcc}
\hline \multicolumn{1}{c}{ TEMA PRINCIPAL } & INCIDÊNCIA & \% \\
\hline NACIONAL & & \\
Empreendedorismo & 05 & 20,8 \\
Estratégia & 05 & 20,8 \\
Internacionalização & 04 & 16,6 \\
Contratos & 03 & 12,5 \\
Conflitos & 02 & 8,3 \\
Gestão social & 02 & 8,3 \\
Marketing & 01 & 4,1 \\
Finanças & 01 & 4,1 \\
Padronização & 01 & 4,1 \\
TOTAL & $\mathbf{2 4}$ & $\mathbf{1 0 0 , 0}$ \\
\hline
\end{tabular}

\section{INTERNACIONAL}

\begin{tabular}{llc} 
Teoria da Agência & 09 & 24,3 \\
Estratégia & 05 & 13,5 \\
Custos de Transação Econômica & 03 & 8,1 \\
Resource Based View [RBV] & 02 & 5,4 \\
Expansão & 02 & 5,4 \\
Relacionamentos & 02 & 5,4 \\
Conflitos & 02 & 5,4 \\
Escassez de Recursos & 01 & 2,7 \\
Tecnologia da Informação & 01 & 2,7 \\
Percepção Pública & 01 & 2,7 \\
Gestão do Conhecimento & 01 & 2,7 \\
Marcas & 01 & 2,7 \\
Estudos Organizacionais & 01 & 2,7 \\
Ética & 01 & 2,7 \\
Marketing & 01 & 2,7 \\
Direitos de Propriedade & 01 & 2,7 \\
Performance & 01 & 2,7 \\
Contratos & 01 & 2,7 \\
Publicidade & 01 & 2,7 \\
TOTAL & $\mathbf{3 7}$ & $\mathbf{1 0 0 , 0}$ \\
\hline
\end{tabular}

Nota. Fonte: elaborado pelos autores. 


\section{Filiação dos Autores}

Buscando maior síntese da relação de filiação dos autores, foi considerada somente a principal filiação do primeiro autor. Nestes estudos, autores vinculados às instituições de ensino com boas avaliações pela CAPES apresentam mais de um artigo publicado sobre franchising, como é o caso da EAESP-FGV/SP (Nota 6); FEA-USP (Nota 6); COPPEAD-UFRJ (Nota 5); CCSA-MACKENZIE/SP (Nota 5); IAG-PUC/RJ (Nota 5) e FEA-RP (Nota 4), correspondendo a cerca de 55\% dos analisados. Os restantes trabalhos se encontram disseminados por outras onze instituições de ensino, o que demonstra a preocupação pela pesquisa científica em administração por diversas instituições.

Em se tratando dos estudos internacionais, nota-se grande pulverização, já que $70 \%$ destes estão vinculados a 26 instituições de ensino, com um trabalho publicado cada, o que demonstra mais o interesse particular destes pesquisadores, comprovando a inexistência de centros consolidados de estudos sobre franchising. Há que destacar a mobilidade de filiação apresentada por alguns autores, acarretando menor número de registros para cada instituição.

Tabela 5

\section{Filiação dos Autores}

\begin{tabular}{lcc}
\hline \multicolumn{1}{c}{ INSTITUIÇÃO } & INCIDÊNCIA & \% \\
\hline NACIONAL & & \\
EAESP (FGV/SP) & 03 & 12,5 \\
FEA (USP) & 02 & 8,3 \\
COPPEAD (UFRJ) & 02 & 8,3 \\
CCSA (MACKENZIE/SP) & 02 & 8,3 \\
IAG (PUC/RJ) & 02 & 8,3 \\
FEA-RP (USP) & 02 & 8,3 \\
FZEA (USP) & 01 & 4,1 \\
FCAV (UNESP) & 01 & 4,1 \\
CCSA (FURB/SC) & 01 & 4,1 \\
IBMEC/SP & 01 & 4,1 \\
EBAPE (FGV/RJ) & 01 & 4,1 \\
CCSA (UNIOESTE/PR) & 01 & 4,1 \\
ICEG (PUC/MG) & 01 & 4,1 \\
FACE (PUC/RS) & 01 & 4,1 \\
CCSH (UFSM/RS) & 01 & 4,1 \\
CFH (UFSC) & 01 & 4,1 \\
CSA (UEM/PR) & 01 & 4,1 \\
TOTAL & $\mathbf{2 4}$ & $\mathbf{1 0 0 , 0}$ \\
\hline
\end{tabular}

INTERNACIONAL
U. of Illinois/EUA
03
8,1
U. of California/EUA
02
5,4 


\section{Tabela 5 (continuação)}

\begin{tabular}{|c|c|c|}
\hline INSTITUIÇÃO & INCIDÊNCIA & $\%$ \\
\hline Florida State U./EUA & 02 & 5,4 \\
\hline U. of Viena/AUT & 02 & 5,4 \\
\hline U. of Las Palmas/ESP & 01 & 2,7 \\
\hline U. of Ulster/UK & 01 & 2,7 \\
\hline Purdue U./EUA & 01 & 2,7 \\
\hline U. of Surrey/UK & 01 & 2,7 \\
\hline U. of Pennsylvania/EUA & 01 & 2,7 \\
\hline Salisbury U./EUA & 01 & 2,7 \\
\hline U. of Queensland/AUS & 01 & 2,7 \\
\hline State U. of New York/EUA & 01 & 2,7 \\
\hline U. of Dayton/EUA & 01 & 2,7 \\
\hline Manchester School of Management/UK & 01 & 2,7 \\
\hline Case Western Reserve U./EUA & 01 & 2,7 \\
\hline Lulea U. of Technology/EUA & 01 & 2,7 \\
\hline U. of Tasmania/AUS & 01 & 2,7 \\
\hline U. of Southern California/EUA & 01 & 2,7 \\
\hline U. of Montana/EUA & 01 & 2,7 \\
\hline U. of Delaware/EUA & 01 & 2,7 \\
\hline Memorial U. of Newfoundland/EUA & 01 & 2,7 \\
\hline U. of Memphis/EUA & 01 & 2,7 \\
\hline U. of Kentucky/EUA & 01 & 2,7 \\
\hline Claremont Mckenna College/EUA & 01 & 2,7 \\
\hline U. of Paris I/FRA & 01 & 2,7 \\
\hline Iowa State U./EUA & 01 & 2,7 \\
\hline Massachusetts Institute Technology/EUA & 01 & 2,7 \\
\hline Christopher Newport U./EUA & 01 & 2,7 \\
\hline U. of South Florida/EUA & 01 & 2,7 \\
\hline Boston U./EUA & 01 & 2,7 \\
\hline Sem Identificação & 02 & 5,4 \\
\hline TOTAL & 37 & 100,0 \\
\hline
\end{tabular}

Nota. Fonte: elaborado pelos autores.

\section{Publicação por Região}

A produção nacional sobre franchising está representada em sua totalidade por estados pertencentes às regiões Sudeste e Sul, levando-se em consideração a filiação do primeiro autor. Estados com maiores participações no PIB nacional, São Paulo e Rio de Janeiro representam 70\% desta produção científica; deste valor, $50 \%$ são de autores filiados às instituições paulistas de ensino. 
Internacionalmente, a origem destas produções científicas procede de países desenvolvidos, sendo os Estados Unidos responsáveis por cerca de $65 \%$ das autorias. Autores de nações européias estão presentes em aproximadamente 22\% destes trabalhos. Estes parâmetros nos levam a crer que há um incentivo maior às pesquisas em estados brasileiros mais desenvolvidos e internacionalmente em nações que gozam de maior poderio econômico.

Tabela 6

Publicação por Região

\begin{tabular}{lcc}
\hline \multicolumn{1}{c}{ REGIÃO } & INCIDÊNCIA & \% \\
\hline NACIONAL & & \\
São Paulo & 12 & 50,0 \\
Rio de Janeiro & 05 & 20,8 \\
Paraná & 02 & 8,3 \\
Santa Catarina & 02 & 8,3 \\
Rio Grande do Sul & 02 & 8,3 \\
Minas Gerais & 01 & 4,1 \\
TOTAL & $\mathbf{2 4}$ & $\mathbf{1 0 0 , 0}$ \\
\hline
\end{tabular}

\section{INTERNACIONAL}

\begin{tabular}{llc} 
Estados Unidos & 24 & 64,8 \\
Reino Unido & 03 & 8,1 \\
Austrália & 03 & 8,1 \\
Austria & 02 & 5,4 \\
Espanha & 01 & 2,7 \\
França & 01 & 2,7 \\
Suécia & 01 & 2,7 \\
Sem identificação & 02 & 5,4 \\
TOTAL & $\mathbf{3 7}$ & $\mathbf{1 0 0 , 0}$ \\
\hline
\end{tabular}

Nota. Fonte: elaborado pelos autores.

\section{Publicação por Pesquisador}

A produção em parceria pode ser notada tanto em artigos internacionais como nacionais; em ambos os casos, estas parcerias ocorrem prioritariamente entre dois autores. Esta configuração é presente em cerca de $42 \%$ dos artigos nacionais e $62 \%$ internacionalmente. A segunda opção tanto nacionalmente como internacionalmente são as produções independentes que constituem aproximadamente $21 \%$ da produção nacional e $30 \%$ da internacional. 
Tabela 7

Autores por Artigos

\begin{tabular}{|c|c|c|}
\hline NÚMERO DE AUTORES & ARTIGOS & $\%$ \\
\hline \multicolumn{3}{|l|}{ NACIONAL } \\
\hline 1 & 05 & 20,8 \\
\hline 2 & 10 & 41,6 \\
\hline 3 & 06 & 25,0 \\
\hline 4 & 02 & 8,3 \\
\hline 5 & 01 & 4,1 \\
\hline TOTAL & 24 & 100,0 \\
\hline
\end{tabular}

INTERNACIONAL

1

2

3

4

5
11

23

02

01

00

37
29,7

62,1

5,4

2,7

0,0

TOTAL

100,0

Nota. Fonte: elaborado pelos autores.

A produção por autor, que leva em consideração a condição de co-autoria, demonstra que nacionalmente o máximo de artigos publicados sobre franchising não ultrapassou a marca de dois artigos, deixando sinais de não haver pesquisadores centrados nestes estudos, estando a produção dispersa em 96\% dos casos em autores que somente obtiveram uma publicação nos veículos analisados. Internacionalmente, cerca de $15 \%$ dos autores possuem mais de uma publicação, encontrando-se cinco autores com dois artigos, dois autores com três artigos e somente um autor com quatro artigos. Estes dados sinalizam uma maior continuidade nos estudos sobre franchising por uma ainda restrita comunidade de pesquisadores internacionais.

Tabela 8

\section{Artigos por Autor}

\begin{tabular}{lccc}
\hline \multicolumn{1}{c}{ AUTORES } & NÚMERO DE AUTORES & $\mathbf{\%}$ & PRINCIPAIS AUTORES \\
\hline NACIONAL & & & \\
Com 1 artigo & 52 & 96,2 & --- \\
Com 2 artigos & 02 & 3,7 & Hoeltebaum, Marianne \\
& & & Marques, Dalton \\
TOTAL & $\mathbf{5 4}$ & $\mathbf{1 0 0 , 0}$ & \\
\hline
\end{tabular}

Continua 
Tabela 8 (continuação)

\begin{tabular}{lccc}
\hline \multicolumn{1}{c}{ AUTORES } & NÚMERO DE AUTORES & $\mathbf{\%}$ & PRINCIPAIS AUTORES \\
\hline INTERNACIONAL & & & --- \\
Com 1 artigo & 46 & 85,1 & Hoffman, Richard \\
Com 2 artigos & 05 & 9,2 & Kaufman, Patrick \\
& & & Kleiner, Brian \\
& & & Preble, John \\
& & & Shane, Scott \\
& 02 & 3,7 & Combs, James \\
Com 3 artigos & & & Dant, Rajiv \\
& & & Michael, Steven \\
Com 4 artigos & 01 & 1,8 & \\
TOTAL & $\mathbf{5 4}$ & $\mathbf{1 0 0 , 0}$ & \\
\hline
\end{tabular}

Nota. Fonte: elaborado pelos autores.

\section{Método de Pesquisa}

A preocupação por trabalhos quantitativos é evidente nos trabalhos nacionais sobre franchising, em cerca de $42 \%$ destes há emprego de surveys. Internacionalmente este método é ainda mais expressivo, apresentando-se em $62 \%$ dos artigos analisados. Isto comprova o rigor quanto ao emprego de técnicas estatísticas presentes em veículos científicos de primeira linha, menos em artigos nacionais e mais em artigos internacionais, o que demonstra até mesmo o alinhamento do meio acadêmico brasileiro com o exterior.

Entretanto o método do estudo de caso possui significativa participação nos trabalhos nacionais, constituindo $46 \%$ das publicações, fazendo-se presente em somente $8 \%$ dos trabalhos internacionais. Artigos de discussão teórica assumem uma parcela mais significativa internacionalmente do que os estudos de caso, constituindo $24 \%$ destas publicações.

Tabela 9

Método de Pesquisa

\begin{tabular}{lcc}
\hline \multicolumn{1}{c}{ MÉTODO } & INCIDÊNCIA & \% \\
\hline NACIONAL & & \\
Estudo de Casos & 11 & 45,8 \\
Survey & 10 & 41,6 \\
Discussão Teórica & 02 & 8,3 \\
Etnográfico & 01 & 4,1 \\
TOTAL & $\mathbf{2 4}$ & $\mathbf{1 0 0 , 0}$ \\
\hline
\end{tabular}




\section{Tabela 9 (continuação)}

\begin{tabular}{lcc}
\hline \multicolumn{1}{c}{ MÉTODO } & INCIDÊNCIA & \% \\
\hline INTERNACIONAL & & \\
Survey & 23 & 62,1 \\
Discussão Teórica & 09 & 24,3 \\
Estudo de Casos & 03 & 8,1 \\
Meta-Análise & 01 & 2,7 \\
Análise de Conteúdo & 01 & 2,7 \\
TOTAL & $\mathbf{3 7}$ & $\mathbf{1 0 0 , 0}$ \\
\hline
\end{tabular}

Nota. Fonte: elaborado pelos autores.

\section{Segmento Econômico}

O segmento econômico, tratado nestes estudos, tanto nacionais, em $29 \%$ dos casos, como internacionais, em $62 \%$ dos artigos, não trata de segmentos específicos. Estes números se justificam, em grande parte, por surveys que não restringem suas coletas de dados por setores econômicos. Por sua ordem, o setor de Alimentação foi o que mais se apresentou nos estudos nacionais em $29 \%$ dos artigos e internacionais em $22 \%$ destas publicações.

Tabela 10

\section{Segmento Econômico}

\begin{tabular}{lcc}
\hline \multicolumn{1}{c}{ SETOR } & INCIDÊNCIA & $\mathbf{\%}$ \\
\hline NACIONAL & 07 & 29,1 \\
Não específico & 07 & 29,1 \\
Alimentação & 02 & 8,3 \\
Beleza & 02 & 8,3 \\
Educação & 01 & 4,1 \\
ONG & 01 & 4,1 \\
Vestuário & 01 & 4,1 \\
Pet Shop & 01 & 4,1 \\
Imobiliário & 01 & 4,1 \\
Governo & 01 & 4,1 \\
Correspondência & $\mathbf{2 4}$ & $\mathbf{1 0 0 , 0}$ \\
TOTAL & &
\end{tabular}

\section{INTERNACIONAL}

Não específico

Alimentação
23

08
62,1

21,6

\section{Continua}


Tabela 10 (continuação)

\begin{tabular}{lcc}
\hline \multicolumn{1}{c}{ SETOR } & INCIDÊNCIA & \% \\
\hline Hospedagem & 02 & 5,4 \\
Esportivo & 02 & 5,4 \\
Transporte público & 01 & 2,7 \\
Vestuário & 01 & 2,7 \\
TOTAL & $\mathbf{3 7}$ & $\mathbf{1 0 0 , 0}$ \\
\hline
\end{tabular}

Nota. Fonte: elaborado pelos autores.

\section{CONSIDERAÇÕES FINAIS}

Neste estudo, em que se realizou um levantamento da produção científica nacional e internacional sobre franchising, foi possível obter uma proporção eqüitativa entre artigos nacionais (40\%) e internacionais (60\%). Entretanto estes números absolutos são modestos. Ao se considerar que somente no último Encontro Nacional da Associação Nacional de Pós-Graduação e Pesquisa em Administração [ENANPAD] (2007) houve 982 artigos aceitos e que no período de 10 anos houve somente 24 artigos sobre franchising, pode-se notar que se trata de uma temática pouco debatida pela academia brasileira.

Por sua vez, este setor é economicamente importante, sendo responsável pela movimentação de mais de R 46 bilhões em 2007, além de apresentar um índice de crescimento marcante, superior a 15\% no último ano (ABF, 2008).

Cumpre destacar que parte deste reduzido número de trabalhos selecionados se deu devido ao rigor metodológico adotado nesta pesquisa. Somente participaram artigos vinculados ao website da Anpad, por ser este um aglutinador tanto de eventos como periódicos bem avaliados pelo sistema Qualis; e artigos internacionais presentes em periódicos classificados como Internacionais A, B ou C disponíveis no portal Proquest.

O Enanpad, principal evento da área, representou $80 \%$ dos artigos analisados nacionalmente. Internacionalmente, os periódicos Strategic Management Journal, European Journal of Marketing e Journal of Retailing concentram 43\% destas publicações, caracterizando periódicos que tratam de abordagens estratégicas e mercadológicas.

É importante enunciar que veículos científicos atrelados à Anpad não registraram nenhuma ocorrência de publicações nos últimos anos, como é o caso dos periódicos RAE, RAC, BAR, RAC-E e dos eventos ENEO, ENAPG, ENGPR, ENADI e ENEPQ. Parte desta ausência de publicações sobre franchising pode ser explicada pelo caráter ainda recente destes eventos. Dois fatores contribuíram para a limitação do número de artigos analisados; um deles é o número de veículos científicos e o outro, a linha temporal, restrita aos anos compreendidos entre 1998 e 2007. Caso fossem estendidos estes critérios, certamente se teria maior número de artigos na base analisada.

Quanto à disposição destes trabalhos ao longo dos anos, notou-se uma continuidade, excetuando o ano 2002. Nos dois últimos anos, 2006 e 2007, houve participação maior dos trabalhos sobre franchising no Brasil, o que representa cerca de um terço da produção do período analisado. Este indicador pode sinalizar o meio acadêmico, quanto ao despertar pelos estudos sobre franchising; porém somente por meio de uma análise estatística minuciosa poderíamos comprovar esta tendência.

Nacionalmente a presença de estudos sobre franchising que envolvam Empreendedorismo e Estratégia é marcante, representando mais de $40 \%$ deles. Isto pode demonstrar a preocupação destes pesquisadores em analisar o franchising sob a ótica do empreendedorismo e seu cunho estratégico. Por 
sua vez, internacionalmente, os estudos mais marcantes se localizam em teorias econômicas, como a Teoria da Agência, Custos de Transação, Resource Based View e Escassez de Recursos que representam 38\% destes trabalhos. Estas temáticas podem indicar novos caminhos aos pesquisadores brasileiros na busca de estudos mais sistemáticos sobre o sistema de franchising.

Pesquisadores brasileiros filiados a programas de pós-graduação stricto sensu bem avaliados detêm cerca de 55\% destas autorias, o que indica o comprometimento destas instituições com o processo de avaliação institucionalizado pela CAPES. Internacionalmente há grande pulverização entre as instituições, em que 70\% das autorias são representadas por 26 instituições de ensino. Isto caracteriza, principalmente nos estudos internacionais, a inexistência de polos de excelência em estudos sobre franchising.

O Brasil, por ser país continental, tem no franchising um modelo adequado para a expansão de muitas empresas; entretanto, dentre os artigos analisados, houve uma concentração de trabalhos oriundos das regiões Sul e Sudeste; o Estado de São Paulo foi responsável por 50\% desta produção. Internacionalmente a presença dos Estados Unidos é notória em 65\% destas autorias; os Estados Unidos, aliás, são também o maior país em número de franquias. Estes dados indicam que regiões mais favorecidas economicamente são também aquelas que empenham maiores recursos acadêmicos no estudo de sua atividade econômica.

Com relação à parceria para produção científica, isto ocorre intensamente tanto nacionalmente, em 80\% dos artigos; como internacionalmente, em $70 \%$ destes. A preferência de parceria entre dois autores por artigo está em $42 \%$ dos trabalhos nacionais e em $62 \%$ dos internacionais. Tais resultados demonstram a reconhecida importância da troca de informações e conhecimentos para a elaboração de publicações em veículos científicos em que há rigorosas avaliações.

Quando se trata da publicação por autor, nota-se que nacionalmente há poucos autores que dão continuidade aos seus trabalhos sobre franchising; somente dois autores possuíram mais de uma autoria. Este não é um indicador favorável, pois demonstra uma falta de continuidade nestes estudos, o que poderia ser benéfico ao setor. Já internacionalmente, encontra-se um grupo de oito autores que possuem mais de dois artigos publicados, demonstrando que há uma continuidade maior nos estudos sobre franchising.

A academia brasileira acompanha a prática metodológica internacional no emprego de estudos quantitativos. Há presença de surveys em $42 \%$ dos estudos nacionais; esta mesma metodologia encontra-se em $62 \%$ dos estudos internacionais. Os estudos de caso foram mais representativos nos estudos nacionais, sendo utilizados em $46 \%$ das pesquisas; por sua vez, a adoção internacional desta metodologia é mais restrita; em somente $24 \%$ dos artigos há o seu emprego. Os surveys são mais abrangentes e conseguem gerar considerações mais amplas sobre as populações observadas do que os estudos de caso, que buscam tratar de situações particularizadas (Babbie, 1999; Yin, 2001).

Dentre as variáveis estudadas, nota-se tanto nos estudos nacionais quanto internacionais o não direcionamento da aplicação destas pesquisas em setores econômicos em $29 \%$ dos artigos nacionais e $62 \%$ internacionalmente. Dados que podem ser compreendidos por meio da intensa utilização de surveys que aglomeram franquias de diversos setores. Por sua vez, o setor de alimentação foi o que teve o maior destaque em ambos os casos: $29 \%$ dos estudos nacionais e em $22 \%$ dos estudos internacionais.

Por fim, o Brasil representa atualmente a quarta posição mundial em números de unidades franqueadas; contudo a sua participação em periódicos internacionais indexados ao sistema Qualis não apresentou nenhum trabalho nos últimos 10 anos. Este indicador, pouco animador em um primeiro momento, talvez possa servir de incentivo aos pesquisadores interessados no sistema de franchising e mesmo em formas de empreendedorismo. Pode-se considerar que em campo econômico tão representativo na economia brasileira, ainda há vasto território para a exploração dos pesquisadores brasileiros, que podem contar com campo de estudo que apresenta cerca 1.197 redes de franquias e 65.500 unidades franqueadas (ABF, 2008). 
A título de sugestões de pesquisas futuras, estudos mais aprofundados sobre os indicadores apresentados podem ser empregados, além de envolver maior número de veículos científicos, linha temporal mais extensa e até mesmo o desenvolvimento de teorias ainda pouco exploradas nacionalmente.

Artigo recebido em 11.09.2008. Aprovado em 06.03.2009.

\section{REFERÊNCIAS BIBLIOGRÁFICAS}

Almeida, S., Lopes, T., \& Pereira, R. (2006, maio). A produção científica em marketing de relacionamentos no Brasil entre 1990 e 2004. Anais do Encontro de Marketing, Rio de Janeiro, RJ, Brasil, 2.

Associação Brasileira de Franchising (2008). Desempenho 2007 - 2008. Recuperado em 5 março, 2008, de http://www.portaldofranchising.com.br/site/content/interna/index.asp?codA=11\&codC=10\&origem=s obreosetor

Azevedo, P., \& Silva, A. (2003, outubro). Entre a autoridade e a autonomia: as gerações de franchising. Proceedings of the International Conference on Economics and Agri-Food Networks Management, New York, NY, USA, 4.

Babbie, E. (1999). Métodos de pesquisas de survey. Belo Horizonte: Editora UFMG.

Caldas, M., Tonelli, M., \& Lacombe, B. (2002, setembro). Espelho, espelho meu: meta-estudo da produção científica em recursos humanos nos Enanpads da década de 90. Anais do Encontro Nacional da Associação Nacional de Pós-Graduação e Pesquisa em Administração, Salvador, BA, Brasil, 26.

Canzian, F., \& Lage, J. (2008, março 13). Investimentos e mercado interno puxam economia. Folha de São Paulo, Recuperado em 15 março, 2008, de http://www1.folha.uol.com.br/fsp/dinheiro/fi1303200802.htm

Cardoso, R., Pereira, C., \& Guerreiro, R. (2007). Perfil das pesquisas em contabilidade de custos apresentados no Enanpad no período de 1998 a 2003. Revista de Administração Contemporânea, 11(3), 177-198.

Closs, L., Aramburú, J., \& Antunes, E. (2006, setembro). Produção científica sobre o ensino em administração: uma avaliação envolvendo o enfoque do paradigma da complexidade. Anais do Encontro Nacional da Associação Nacional de Pós-Graduação e Pesquisa em Administração, Salvador, BA, Brasil, 30.

Combs, J., \& Ketchen, D., Jr. (2003). Why do firms use franchising as an entrepreneurial strategy? A meta-analysis. Journal of Management, 29(3), 443-465.

Correa, P., Holtegebaum, M., \& Machado, H. (2006, outubro). Análise do perfil empreendedor dos franqueados de escolas de idiomas na cidade de Londrina, Paraná. Anais do Simpósio de Gestão da Inovação Tecnológica, Gramado, RS, Brasil, 24.

Dant, R., Paswan, A., \& Kaufman, P. (1996). What we know about ownership redirection in franchising: a meta-analysis. Journal of Retailing, 72(4), 429-445.

Dornelas, J. (2001). Empreendedorismo: transformando idéias em negócios. Rio de Janeiro: Elsevier.

Durante, D., \& Maurer, S. (2007, setembro). Gestão do conhecimento e da informação: revisão da produção científica do período 2000-2005. Anais do Encontro Nacional da Associação 
Nacional de Pós-Graduação e Pesquisa em Administração, Rio de Janeiro, RJ, Brasil, 31.

Encontro Nacional da Associação Nacional de Pós-Graduação e Pesquisa em Administração (2007, setembro). Resumo dos trabalhos. Rio de Janeiro, RJ: Autor.

Geyskens, I., Steenkamp, J., \& Kumar, N. (1999). A meta-analysis of satisfaction in marketing channel relationship. Journal of Marketing Research, 36(1), 123-138.

Hisrich, R., \& Peters, M. (2004). Empreendedorismo. Porto Alegre: Bookman.

Hitt, M., Ireland, R., \& Hoskisson, R. (2002). Administração estratégica - Competitividade e globalização. São Paulo: Pioneira Thomson Learning.

Hocayen-da-Silva, A., Rossoni, L., \& Ferreira, I., Jr. (2006, novembro). Administração pública e gestão social: a produção científica brasileira entre 2000 e 2005. Anais do Encontro de Administração Pública e Governança, São Paulo, SP, Brasil, 2.

Hoppen, N., Audy, J., Zanela, A., Candotti, C., Santos, A., Scheid, R., Perin, M., Mecca, M., \& Petrini, M. (1998, setembro). Sistemas de informação no Brasil: uma análise dos artigos científicos dos anos 90. Anais do Encontro Nacional da Associação Nacional de PósGraduação e Pesquisa em Administração, Foz do Iguaçu, PR, Brasil, 27.

Horngren, C. (1989). Cost and management accounting: yesterday and today. Journal of Management Accounting Research, 3(1), 21-32.

International Franchise Association (2008). How big is franchising? Recuperado em 8 janeiro, 2008, de http://www.franchise.org/Franchise-News-Detail.aspx?id=34192

Luiz, D., Motoki, L., Villela, J., Ura, I., \& Lourenzani, A. (2006, setembro). Franchising como forma de negócio: um estudo preliminar no município de Tupã (SP). Anais do Encontro Nacional da Associação Nacional de Pós-Graduação e Pesquisa em Administração, Salvador, BA, Brasil, 30.

Machado-da-Silva, C., Cunha, V., \& Amboni, N. (1990, setembro). Organizações: o estado da arte da produção acadêmica. Anais do Encontro Nacional da Associação Nacional de Pós-Graduação e Pesquisa em Administração, Florianópolis, SC, Brasil, 14.

Matias, M., Machado, M., Machado, M. A., \& Martins, G. (2007, novembro). Análise epistemológica da produção científica em contabilidade sob a ótica da estruturação interna. Anais do Encontro de Ensino e Pesquisa em Administração e Contabilidade, Recife, PE, 1.

Melo, P., Borini, F., \& Ribeiro, F. (2007, agosto). Em busca de delimitações do enquadramento do franchising como alianças estratégicas. Anais dos Seminários em Administração, São Paulo, SP, Brasil, 10.

Otchere, M. (2003). Accounting and finance at forty: a retrospective evaluation. Accounting \& Finance, 43(2), 211-231.

Qualis. (2007). Webqualis. Recuperado em 1 outubro, 2007, de http://qualis.capes.gov.br/webqualis/ConsultaListaCompletaPeriodicos.faces

Rizzo, M. (2005). Franchise, o negócio do século. São Paulo: Rizzo Franchise.

Rossoni, L., Ferreira, I., Jr., \& Hocayen-da-Silva, A. (2006, outubro). Administração de ciência e tecnologia: a produção científica brasileira entre 2000 e 2005. Anais do Simpósio de Gestão da Inovação Tecnológica, Gramado, RS, Brasil, 24.

Rossoni, L., Guarido, E., Franscisconi, K., \& Albuquerque, J. (2007, maio). Estratégia em organizações: a produção científica em eventos nacionais entre 2001 e 2006. Anais do Encontro de Estudos em Estratégia, São Paulo, SP, Brasil, 3. 
Rossoni, L., \& Hocayen-da-Silva, A. (2007, outubro). Administração da informação: a produção científica brasileira entre 2001 e 2006. Anais do Encontro de Administração da Informação, Florianópolis, SC, Brasil, 1.

Shane, S. (2005). From ice cream to the internet: using franchising to drive the growth and profits of your company. Upper Saddler River, NJ: Prentice Hall.

Shields, M. (1997). Research in management accouting by north americans in the 1990s. Journal of Management Accounting Research, 9(1), 3-60.

Silva, R., \& Campos, L., Filho (2007, maio). Evolução da produção científica brasileira em gestão internacional entre 1997 e 2006. Anais do Encontro de Estudos em Estratégia, São Paulo, SP, Brasil, 3.

Swartz, L. (2001). Franchising successfully circles the globe. In D. H. B. Welsh \& I. Alon (Eds.), International franchising in emerging markets: central and eastern Europe and Latin America (pp. 43-61). Chicago: CCH.

Szymanski, D., Bharadwaj, S., \& Varadarajan, P. (1993). An analysis of the market share-profitability relationship. Journal of Marketing, 57(1), 1-18.

Tonelli, M., Caldas, M., Lacombe, B., \& Tinoco, T. (2003). Produção acadêmica em recursos humanos no Brasil: 1991-2000. Revista de Administração de Empresas, 43(1), 104-122.

Vieira, F. (1998, setembro). Por quem os sinos dobram? Uma análise da publicação científica na área de marketing do ENANPAD. Anais do Encontro Nacional da Associação Nacional de PósGraduação e Pesquisa em Administração, Foz do Iguaçu, PR, Brasil, 22.

Vollmers, G. (1996). Academic cost accouting from 1920-1950: alive and well. Journal of Management Accounting Research, 8(1), 183-199.

Wood, T., Jr., \& Chueke, G. (2007, novembro). Ranking de produção científica em administração de empresas no Brasil. Anais do Encontro de Ensino e Pesquisa em Administração $e$ Contabilidade, Recife, PE, Brasil, 1.

Yin, R. (2001). Estudo de caso: planejamento e métodos. Porto Alegre: Bookman.

Zimmer, M., \& Leis, R. (2007, outubro). Estado-da-arte sobre a produção científica brasileira em gestão do conhecimento: um estudo em periódicos nacionais e nos anais do Enanpad no período 1997-2006. Anais do Encontro de Administração da Informação, Florianópolis, SC, Brasil, 1. 